\title{
Structure of Bilayer Membranes of Gemini Surfactants with Rigid and Flexible Spacers from MD Simulations
}

\author{
Dmitry Yakovlev ${ }^{1}$ and Edo S. Boek ${ }^{2}$ \\ 1 St.Petersburg State University, Department of Chemistry, \\ 26 Universitetsky pr., 198504 St.Petersburg, Russia \\ 2 Schlumberger Cambridge Research, \\ High Cross, Madingley Road, Cambridge CB3 0EL, United Kingdom, \\ boek@cambridge.oilfield.slb.com
}

\begin{abstract}
Molecular Dynamics simulations were performed for $9 \times 9$ bilayers formed by gemini surfactants $p$ - $\left[\mathrm{C}_{19} \mathrm{H}_{39} \mathrm{~N}^{+}\left(\mathrm{CH}_{3}\right)_{2} \mathrm{CH}_{2}\right]_{2} \mathrm{C}_{6} \mathrm{H}_{4}$. $2 \mathrm{Cl}^{-}$or $\left[\mathrm{C}_{19} \mathrm{H}_{39} \mathrm{~N}^{+}\left(\mathrm{CH}_{3}\right)_{2} \mathrm{CH}_{2}\right]_{2} \mathrm{CH}(\mathrm{OH}) \cdot 2 \mathrm{Cl}^{-}$with rigid hydrophobic or flexible hydrophilic types of spacer respectively. The structure of the bilayers is rather different depending on the type of spacer regarding the effect of $\mathrm{NaCl}$ salt addition. It is shown that the structure of the bilayer strongly depends on the interaction between the surfactant head groups and the counter ions.
\end{abstract}

\section{Introduction}

An experimental study of gemini surfactants with different types of spacer has been reported in a number of papers 123 . So-called gemini or bis-surfactants are formed by two novel surface active molecules connected via a flexible or rigid linkage between the hydrophilic head groups. These systems are of interest due to a number of unusual properties. One of these is that the CMC value increases when the hydrocarbon chain of the hydrophobic tail reaches some value. This observation is in contradiction with the well-known fact that the CMC value - in general - monotonically decreases with tail lengthening. It is worth to mention that some novel surfactants with long hydrophobic tail display similar behaviour. To understand the physical backgroud of these unusual micellar properties, Molecular Dynamics (MD) simulations were used.

Molecular Dynamics study is a method to observe the microscopic structure of aggregates and to extract a number of macroscopic parameters to be used in thermodynamic models of micellization. Spontaneous formation of spherical or rodlike micelles (depending on surfactant concentration) from a random initial configuration has been reported in a number of papers [4,67]. Self-organization was observed for systems where the concentration of surfactants exceeds the $\mathrm{CMC}$ value. MD simulation with random starting configuration allows us to estimate the CMC value of a surfactant with a given molecular structure. However, this method cannot be applied to systems with a CMC value below some 
limit due to computational expenses. For example, in the case of a CMC value of $10^{-3} \mathrm{M}$, the number of water molecules per surfactant is greater than 55000 ; for a spherical micelle with average aggregation number about 60 , the size of the system is then too large to observe aggregation in a standard MD simulation starting from a random initial configuration. The reasonable way of MD simulations of such systems is to construct a bicontinuous bilayer surrounded by water [8].

In this work, MD simulations have been performed to compare the properties of bilayers formed by gemini surfactants (see Fig. 1) with rigid and flexible spacers. The length of the hydrocarbon chains in both cases was taken as 19 carbon atoms. To observe the effect of electrolyte addition on the bilayer structure, the simulations were carried out also for systems with 3 percent of $\mathrm{NaCl}$ salt. Overall four systems have been considered. The first one includes gemini surfactant with rigid hydrophobic spacer $\left[\left(\mathrm{C}_{19} \mathrm{H}_{39} \mathrm{~N}^{+}\left(\mathrm{CH}_{3}\right)_{2} \mathrm{CH}_{2}\right)_{2} \mathrm{Ar}\right] \cdot \mathrm{Cl}_{2}^{-}$. We will label this system as "Gemini-Ar". The second system is the same but with 3 percent of added $\mathrm{NaCl}$; the label is "Gemini-Ar-NaCl". The last two systems include a gemini surfactant with flexible hydrophilic spacer $\left[\left(\mathrm{C}_{19} \mathrm{H}_{39} \mathrm{~N}^{+}\left(\mathrm{CH}_{3}\right)_{2} \mathrm{CH}_{2}\right)_{2} \mathrm{CH}(\mathrm{OH})\right] \cdot \mathrm{Cl}_{2}^{-}$. We will refer to these as "Gemini$\mathrm{OH}$ " and "Gemini-OH-NaCl" depending on salt addition.

\section{MD Simulation Details}

The simulations were carried out using GROMACS [9] software running in parallel 10] under MPI environment on an SGI Origin 2000 supercomputer equipped with 32 CPUs. Actually not more than 12 processors were utilized due to communication overhead. The GROMACS united atom force field was used. Equilibrium molecular geometry and atomic charge distributions were taken from semiempirical calculations using the MNDO hamiltonian model. The quantum chemical calculations were performed using the GAMESS 11] program package. The charges of united atom groups $\left(\mathrm{CH}_{n}\right)$ were calculated as the sum of atomic charges over all atoms of given functional group. The major part of the total charge $(+2)$ is found to be distributed over the surfactant head group and the tail hydrocarbon group directly connected to nitrogen. The MOPAC atomic charges on united atoms are given in Fig. 1]

The basic cell has orthogonal geometry with periodic boundary conditions applied in all dimensions. The bilayer was positioned in the middle of the box perpendicular to $z$ axis. The rest of the basic cell was filled with water (SPC model) and ions. To build a bilayer with a density close to the density of liquid hydrocarbons, a special technique was developed. The problem is that - due to the equilibrium molecular geometry of the surfactants under investigation - the constructed bilayer has many cavities between the tails, which are filled with water and ions. The idea is to generate a large number of conformations by running MD simulation of a single surfactant molecule and than find a geometry that provides a suitable density. A number of criteria was used to select the best configuration from formally acceptable ones. The resulting bilayers were 

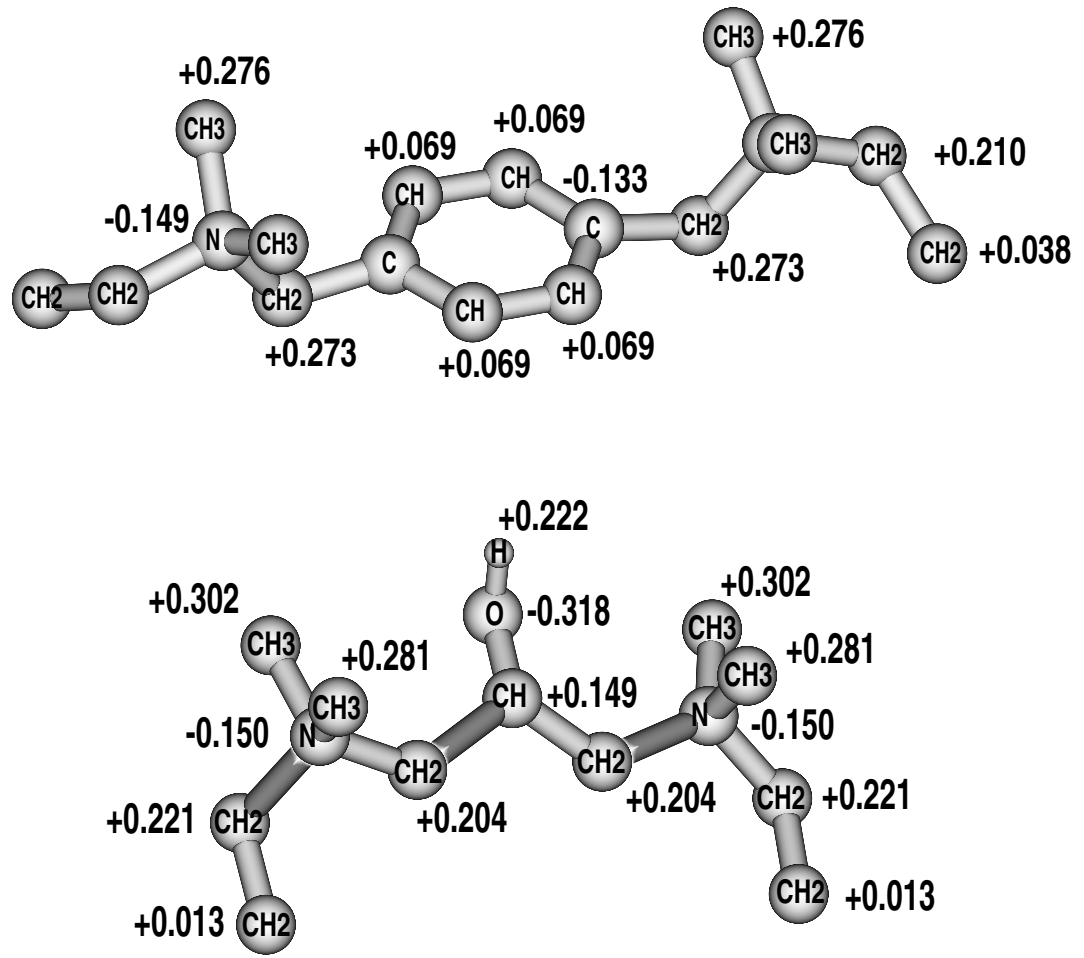

Fig. 1. United atom model and charges of the head groups of gemini surfactants with rigid hydrophobic (top) and flexible hydrophilic (bottom) spacers. Only two carbon atoms of tails are shown (the rest of the tails is cut).

formed by 162 surfactant molecules $(9 \times 9)$ and hardly contain any water inside the hydrophobic core. A summary of all systems studied is presented in Table 1

The MD simulations were performed at a constant temperature of $300 \mathrm{~K}$ and a constant pressure of 1 bar using the Berendsen external bath coupling [12]. To take into account the effect of water penetration inside the bilayer, the basic cell was scaled isotropically in $x$ and $y$ directions but differently in $z$ direction (so-called "semiisotropic" coupling). The bonds were constrained using the SHAKE algorithm and it's special implementation for rigid water molecules (SETTLE) [13]. The leap-frog method with time step 2 fs was used to integrate the equations of motion. A cut-off distance of $1 \mathrm{~nm}$ was applied to all intermolecular interactions. Due to slow penetration of water into the surfactant bilayer, the geometry of basic cell changes. To speed up equilibration, the simulation was split up in two parts: first, long-range electrostatic interactions were cut at 
Table 1. Details of the molecular dynamics simulations. \#Surf and \#W are numbers of surfactant and water molecules, $\# \mathrm{Cl}$ and $\# \mathrm{Na}$ are numbers of chloride and sodium ions and \#Total is total number of atoms in the basic box. $t_{P M E}$ is time of simulation with PME electrostatic summation and $t_{\text {prod }}$ is time used for average production, $p s$.

\begin{tabular}{lrlllllll}
\hline \hline \multirow{2}{*}{ System } & \#Surf & \#W & \#Cl & \#Na & \#Total & $t_{P M E}$ & $t_{\text {prod }}$ \\
\hline Gemini-Ar & 162 & 6163 & 324 & & 27237 & 1160 & 260 \\
Gemini-Ar-NaCl & 162 & 6047 & 392 & 58 & 27005 & 1060 & 310 \\
Gemini-OH & 162 & 6841 & 324 & & 28785 & 1060 & 310 \\
Gemini-OH-NaCl & 162 & 6709 & 390 & 66 & 28521 & 1000 & 500 \\
\hline \hline
\end{tabular}

a distance of $1.0 \mathrm{~nm}$ during $1 \mathrm{~ns}$. This was followed by more than $1 \mathrm{~ns}$ simulation with PME (Particle Mesh Ewald) summation of coulomb interactions [14]. Switching on the PME summation results in a rapid decrease of the cell size in $z$ direction. Equilibrium was defined as the point where the cell geometry becomes stable.

\section{Analysis of MD Data}

The aim of our MD simulation is to clarify the dependence of bilayer structure on the chemical nature of the surfactant and salt addition. The only difference between the surfactants under investigation is the structure of the spacer: rigid hydrophobic in case of Gemini-Ar and flexible hydrophilic in case of Gemini-OH. As will be shown below, the nature of the spacer has a dramatic effect on the behaviour of bilayer.

The equilibrium size of the basic box in $x, y$ and $z$ dimensions provides us with information about the average surface area $a$ per surfactant (see Table 21). Note that we neglect the curvature of the bilayer when calculating the average surface area as $x \cdot y$ divided by number of surfactant in one layer. It is surprising that the surface area per molecule is smaller for Gemini-Ar than for Gemini$\mathrm{OH}$ : obviously the excluded volume of the $\mathrm{CH}_{2} \mathrm{C}_{6} \mathrm{H}_{4} \mathrm{CH}_{2}$ spacer (and therefore the size of the head group) is larger than for $\mathrm{CH}_{2} \mathrm{CH}(\mathrm{OH}) \mathrm{CH}_{2}$ (see Fig. 1). Furthermore, in the case of adding 3 weight per cent $\mathrm{NaCl}$ to the GeminiAr system results in an expansion of the cell in $z$ but squeezing in $x$ and $y$ dimensions. Hence the area per surfactant decreases from $77.7 \AA^{2}$ to $69.9 \AA^{2}$ (see Table 2). The effect of the salt on the geometry of basic box in case of Gemini-OH is quite opposite: the area $a$ increases from $97.7 \AA^{2}$ up to $106.4 \AA^{2}$. These effects can be related with a partial binding of the chloride ions, screening electrostatic repulsion between the charged head groups.

\subsection{Density Profiles}

The number density profiles along the $z$ axis of the systems studied are presented in Fig. 2. These plots demonstrate significant penetration of solution into the 
Table 2. Sizes of the basic box in $x, y$ and $z$ dimentions $(\mathrm{nm})$, density $\rho\left(\mathrm{g} / \mathrm{cm}^{3}\right)$, surface area per one surfactant $a\left(\AA^{2}\right)$, thickness of the bilayer $H(\mathrm{~nm})$ and measure of water penetration into bilayer core $S_{W T}$. See text for details.

\begin{tabular}{lccccccc}
\hline \hline System & $x$ & $y$ & $z$ & $\rho$ & $a$ & $H$ & $S_{W T}$ \\
\hline Gemini-Ar & 7.43 & 8.47 & 6.50 & 0.975 & 77.7 & 3.1 & 770 \\
Gemini-Ar-NaCl & 7.05 & 8.03 & 7.21 & 0.984 & 69.9 & 3.5 & 860 \\
Gemini-OH & 8.27 & 9.57 & 5.23 & 0.982 & 97.7 & 2.4 & 670 \\
Gemini-OH-NaCl & 8.63 & 9.99 & 4.79 & 0.991 & 106.4 & 2.2 & 560 \\
\hline \hline
\end{tabular}

hydrocarbon core of the membrane so that the head groups are surrounded by water. Note that the bilayer formed by Gemini-Ar surfactant has a more diffuse boundary with bulk water than the bilayer formed by Gemini-OH. A sharp water-membrane boundary is typical for ionic surfactants with a negatively charged head group [8]. In the case of gemini surfactants, the boundary is not well-defined, so one needs a way to measure the thickness of the bilayer. It is suitable to determine the thickness of the membrane as the distance between the points where the density profiles of water and hydrocarbon groups cross. In this way the thickness will depend on water penetration into bilayer. The degree of water penetration into the membrane for different systems can be estimated as an overlap integral $S_{W T}$ between the number density profiles of water $n_{W}$ and hydrocarbon groups of the tail $n_{T}$ :

$$
S_{W T}=\int_{0}^{Z} n_{W}(z) n_{T}(z) d z
$$

The overlap integral $S_{W T}$ as well as the thickness $H$ of the membrane are presented in Table 2 The thickness of the bilayer formed by Gemini-OH surfactant is significantly smaller than for Gemini-Ar systems and decreases when adding salt while the thickness of Gemini-Ar bilayer increases. The dependence of the $S_{W T}$ parameter on addition of salt is the same. The density profiles of head groups, chloride and sodium ions (see Fig. 2, bottom) also show that Gemini$\mathrm{OH}$ systems have a more compact structure of the water-bilayer boundary. Note that the head group and chloride ion profiles are very close to each other. This means that the best part of the counter ions is bound to the head groups.

\subsection{Radial Distribution Functions}

The analysis of radial distribution functions (RDF) provides us with more detailed information about the structure of the bilayer. As mentioned above, changing from a rigid hydrophobic to a flexible hydrophilic spacer causes a dramatic distinction in membrane behaviour. The bilayer properties strongly depend on the chemistry of the head group but not on the hydrophobic tail. Interaction of charged heads with ions also plays a key role. Keeping these in mind, we will 
Gemini-Ar
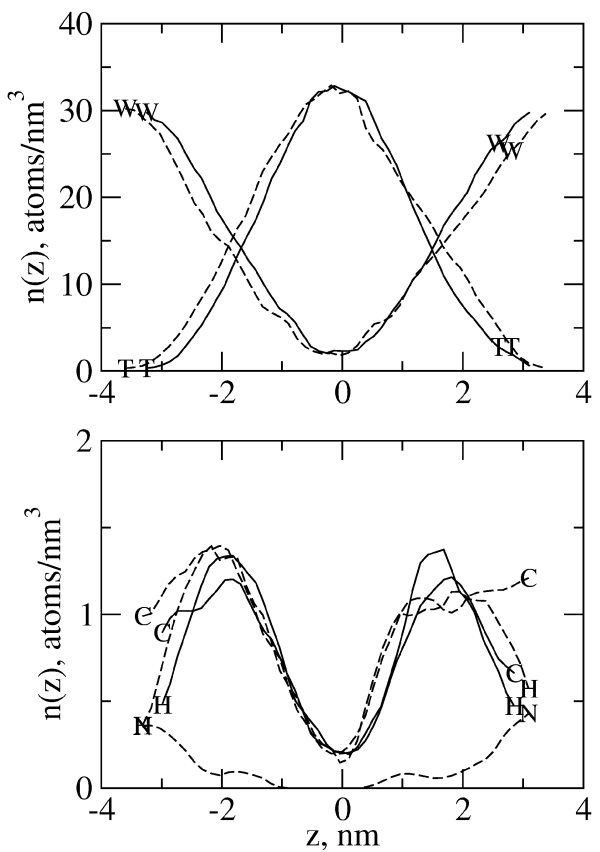

\section{Gemini-OH}
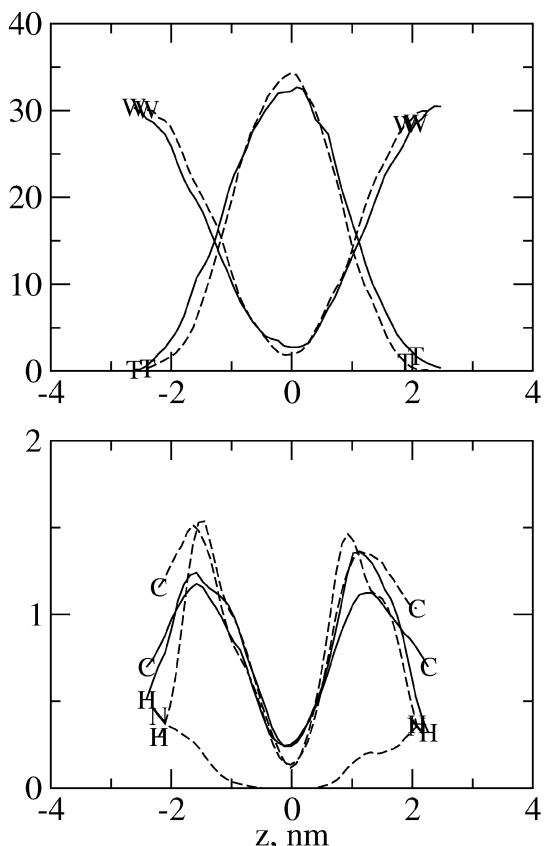

Fig. 2. Number-density profiles across bilayer surface of Gemini-Ar, Gemini-Ar-NaCl (left side) and Gemini-OH, Gemini-OH-NaCl (right side) systems. Systems without salt addition are solid, with salt are dashed. Symbols $W, T, H, C$ and $N$ mark the density profiles of water, hydrocarbon groups of tails, head groups (nitrogen atoms), chloride and sodium ions respectively. Origin of the $z$ axis is shifted to the middle of the basic box for each system. Note that some profiles have different value at left and right edges due to smoothing.

focus our investigation on the set of RDFs related to head-head, head-ions and head-water interaction. The aim is to clarify the origin of the different salt effect on bilayer structure.

The nitrogen-nitrogen radial distribution functions (see Fig. 3, left top) have a well-defined peak that corresponds to two nitrogen atoms of the same surfactant molecule. For Gemini-OH systems this peak is at $r=0.46 \mathrm{~nm}$ while a second maximum at $r=0.86 \mathrm{~nm}$ corresponds with non-bonded nitrogen atoms. In the case of the Gemini-Ar system, these maxima are very close to each other and form one asymmetric peak. The position and magnitude of the peaks of nitrogennitrogen RDFs are almost independent of salt addition (see Table[3). The number of neighbors $N_{c}$ in the first coordination shell was calculated as integral over the RDF up to the first minimum. The nitrogen-nitrogen coordination number for Gemini-Ar is about 10 but only 6.5 in case of Gemini-OH system. This means 
that the bilayer formed by Gemini-Ar surfactants has a diffuse boundary with water because the coordination number is too high for a planar structure. The analysis shows that the positions of the extrema and values of surface area per surfactant for the Gemini-Ar bilayer correspond to hexagonal packing, while the Gemini-OH membrane more likely has an ortorhombic packing. Salt addition causes $N_{c}$ to increase from 9.8 up to 10.2 for Gemini-Ar but to decrease from 6.5 to 6.2 for the Gemini-OH system.

The nitrogen-chloride RDFs demonstrate a strong coupling between chloride ions and head groups. The position of the first maximum remains the same for all systems but the magnitude is subject to change (see Fig. 3, top right and Table 3). Nitrogen has 2.0-2.2 chloride ions in the closest shell for Gemini-Ar and 2.3-2.7 for the Gemini-OH bilayer. Taking into account that each $\mathrm{CH}_{3}$ group of the head has exactly one chloride in the first shell and using molecular geometry, one can conclude that the anions around nitrogen are the ones bound with positively charged $\mathrm{CH}_{3}$ groups connected to nitrogen. The second maximum of the $\mathrm{CH}_{3}$ (head)-chloride RDF (Fig. 3, Table 3) corresponds to chloride of the other $\mathrm{CH}_{3}$ group. In case of the Gemini-OH bilayer, chloride ions are also bound with the $\mathrm{OH}$ functional group. The oxygen-chloride radial distribution function for Gemini-OH (Fig. 3 bottom right) has a sharp first maximum. The benzene ring of Gemini-Ar surfactant has no bound ions as can be observed in the $C H$ (head)chloride RDF (Fig. 3 bottom left) where $\mathrm{CH}$ are the benzene ring atoms (see Fig. 1). The chloride ion connected to the hydroxyl group of Gemini-OH contributes to the first peak of the nitrogen-chloride RDF and the second peak of $\mathrm{CH}_{3}$ (head)-chloride and increases the corresponding coordination numbers. Salt addition always decreases the number of chloride ions in coordination shells because part of chlorides become involved in the formation of ionic pairs with sodium (see Table 3). This is confirmed by the observation that the number of chloride ions in the first coordination shell of sodium (1.3) is greater than one. The positions of the extrema of $\mathrm{CH}_{3}$ (head)-oxygen(water) RDFs are the same for all systems. The number of water molecules in the first hydration shell of the $\mathrm{CH}_{3}$ group is 0.5 only. The reason is that the head groups are surrounded mainly by chloride ions, not by water.

\section{Results and Discussion}

The data presented above demonstrates many distinctions in the structure of bilayers formed by gemini surfactants with different types of spacer. The surfactant with a flexible hydrophilic spacer gives us a thin membrane with a well-defined surface. Changing to a hydrophobic rigid spacer leads to a bilayer with a diffuse boundary. The most interesting question is why salt addition affects the membrane properties in an opposite way. The analysis of radial distribution functions allows us to observe that the interaction between head group and chloride ions controls the behaviour of the bilayer. Packing, bilayer thickness, area per surfactant, water penetration are different for Gemini-Ar and Gemini-OH systems but these properties are secondary. Comparing radial distribution functions for 
Table 3. Positions of maxima $r_{\max }$, minima $r_{\min }(\mathrm{nm})$ and coordination numbers $\mathrm{N}_{c}$ for nitrogen-nitrogen, nitrogen-chloride, $\mathrm{CH}_{3}$ (head)-chloride, $\mathrm{CH}_{3}$ (head)oxygen(water), $C H$ (head)-chlorine (for Gemini-Ar and Gemini-Ar-NaCl) and oxygen(head)-chloride (for Gemini-OH and Gemini-OH-NaCl systems) radial distribution functions (see Fig. 31). The value of $g(r)$ is separated by a slash. In case of well separated first and second maxima, two coordination numbers are given (second coordination number is calculated over both maxima).

\begin{tabular}{|c|c|c|c|}
\hline System & $r_{\max } / g\left(r_{\max }\right)$ & $r_{\min } / g\left(r_{\min }\right)$ & $N_{c}$ \\
\hline \multicolumn{4}{|c|}{ Nitrogen-Nitrogen } \\
\hline Gemini-Ar & $0.80 / 3.9 \quad 0.85 / 1.81 .50 / 1.1$ & $1.24 / 1.0$ & 9.8 \\
\hline Gemini-Ar- $\mathrm{NaCl}$ & $0.80 / 3.9 \quad 0.85 / 1.8 \quad 1.53 / 1.1$ & $1.28 / 1.0$ & 10.2 \\
\hline Gemini-OH & $0.49 / 12.80 .86 / 1.91 .24 / 1.1$ & $1.05 / 1.1$ & 1.26 .5 \\
\hline Gemini-OH-NaCl & $0.49 / 12.80 .86 / 1.91 .25 / 1.1$ & $1.08 / 1.0$ & 1.26 .2 \\
\hline \multicolumn{4}{|c|}{ Nitrogen-Chloride } \\
\hline Gemini-Ar & $0.49 / 4.0 \quad 0.75 / 1.4$ & $0.65 / 1.5$ & 2.2 \\
\hline Gemini-Ar- $\mathrm{NaCl}$ & $0.49 / 3.5 \quad 0.75 / 1.4$ & $0.65 / 1.5$ & 2.0 \\
\hline Gemini-OH & $0.49 / 4.7 \quad 0.70 / 1.7$ & $0.65 / 1.8$ & 2.7 \\
\hline Gemini-OH-NaCl & $0.49 / 4.1 \quad 0.70 / 1.5$ & $0.65 / 1.5$ & 2.3 \\
\hline \multicolumn{4}{|c|}{$\mathrm{CH}_{3}$ (head)-Chloride } \\
\hline Gemini-Ar & $0.39 / 4.1 \quad 0.59 / 2.2$ & $0.51 / 1.7$ & 1.03 .2 \\
\hline Gemini-Ar- $\mathrm{NaCl}$ & $0.39 / 3.8 \quad 0.59 / 2.0$ & $0.51 / 1.6$ & 1.03 .0 \\
\hline Gemini-OH & $0.39 / 4.4 \quad 0.59 / 2.9$ & $0.51 / 1.9$ & 1.13 .7 \\
\hline Gemini-OH-NaCl & $0.39 / 3.9 \quad 0.59 / 2.5$ & $0.51 / 1.7$ & 1.03 .3 \\
\hline \multicolumn{4}{|c|}{$\mathrm{CH}_{3}$ (head)-Oxygen(water) } \\
\hline Gemini-Ar & $0.34 / 2.20 .55 / 1.3$ & $0.47 / 1.1$ & 0.52 .0 \\
\hline Gemini-Ar-NaCl & $0.34 / 2.2 \quad 0.55 / 1.3$ & $0.47 / 1.0$ & 0.42 .0 \\
\hline Gemini-OH & $0.34 / 2.1 \quad 0.55 / 1.4$ & $0.47 / 1.1$ & 0.42 .0 \\
\hline Gemini-OH-NaCl & $0.34 / 2.1 \quad 0.55 / 1.4$ & $0.47 / 1.1$ & 0.52 .1 \\
\hline \multicolumn{4}{|c|}{$C H$ (head)-Chloride } \\
\hline Gemini-Ar & $0.43 / 2.0 \quad 0.65 / 2.1$ & $0.53 / 1.7$ & 0.76 .4 \\
\hline Gemini-Ar-NaCl & $0.43 / 1.9 \quad 0.65 / 2.0$ & $0.53 / 1.5$ & 0.66 .0 \\
\hline \multicolumn{4}{|c|}{ Oxygen(head)-Chloride } \\
\hline Gemini-OH & $0.34 / 3.8 \quad 0.56 / 2.7$ & $0.42 / 1.5$ & 0.46 .5 \\
\hline Gemini-OH-NaCl & $0.34 / 3.3 \quad 0.56 / 2.4$ & $0.42 / 1.2$ & 0.46 .0 \\
\hline
\end{tabular}

surfactants with various types of spacer, one can state that the difference is in the interaction of the head group with chloride ions. The heads of Gemini-OH surfactants coordinate a little bit more chloride ions in the closest shell than Gemini-Ar. The equilibrium thickness of the surfactant membrane is found to be smaller for Gemini-OH than for Gemini-Ar, because the former has a more flexible molecular structure. 

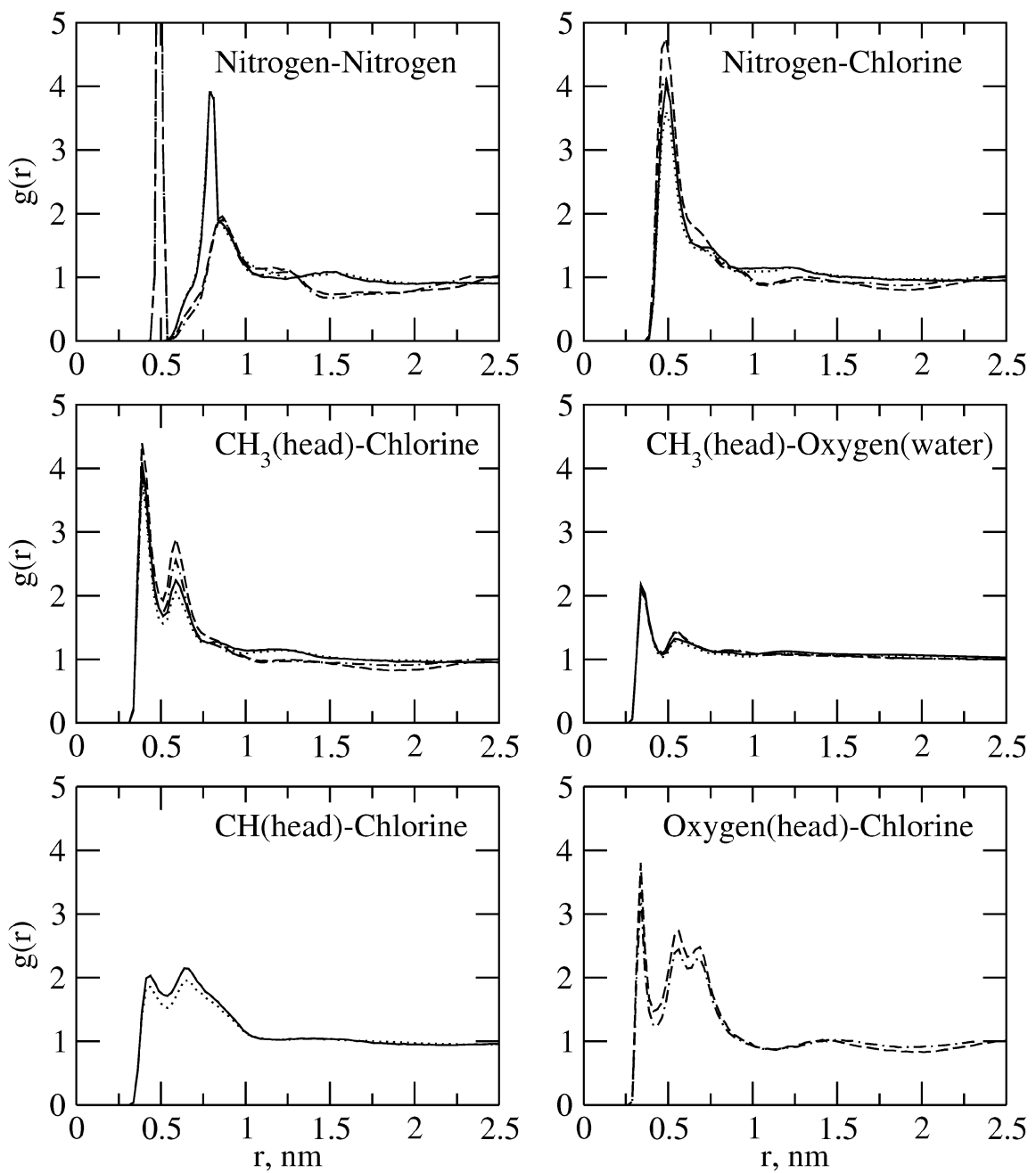

Fig. 3. Nitrogen-nitrogen, nitrogen-chloride, $\mathrm{CH}_{3}$ (head)-chloride, $\mathrm{CH}_{3}$ (head)oxygen(water), $\mathrm{CH}$ (head)-chloride and oxygen(head)-chloride radial distribution functions of Gemini-Ar (solid), Gemini-Ar-NaCl (dotted), Gemini-OH (dashed) and Gemini-OH-NaCl (dash-dotted) systems.

\section{References}

1. Li D. Song and Milton J. Rosen. Surface properties, micellization, and premicellar aggregation of gemini surfactants with rigid and flexible spacers. Langmuir, 12(5):1149-1153, 1996. 
2. Alexander A. Yaroslavov, Oleg Yu. Udalykh, Nickolay S. Melik-Nubarov, Victor A. Kabanov, Yuri A. Ermakov, Vladimir A. Azov, and Fredric M. Menger. Conventional and gemini surfactants embedded with bilayer membranes: contrasting behavior. Chemistry - A European Journal, 7(22):4835-4843, 2001.

3. Fredric M. Menger and Jason S. Keiper. Gemini surfactants. Angewandte Chemie Int. Ed., 39(11):1906-1920, 2000.

4. B. Smit, A. G. Schlijper, L. A. M. Rupert, and N. M. van Os. Effect of chain length of surfactants on the interfacial tension: molecular dynamics simulations and experiments. J. Phys. Chem., 94(18):6933-6935, 1990.

5. B. Smit, P. A. J. Hilbers, K. Esselink, L. A. M. Rupert, N. M. van Os, and A. G. Schlijper. Structure of a water/oil interface in the presence of micelles: a computer simulation study. J. Phys. Chem., 95(16):6361-6368, 1991.

6. Rüdiger Goetz, Gerhard Gompper, and Reinhard Lipowsky. Mobility and elastisity of self-assembled membranes. Phys. Rev. Lett., 82(1):221-224, 1999.

7. S. J. Marrink, D. P. Tielenan, and A. E. Mark. Molecular dynamics simulation of the kinetics of spontaneous micelle formation. J. Phys. Chem. B, 104(51):12165$12173,2000$.

8. E. S. Boek, A. Jusufi, H. Löwen, and G. C. Maitland. Molecular design of responsive fluids: Md studies of viscoelastic surfactant solutions. J.Phys.:Condens.Matter, 14:9413-9430, 2002.

9. E. Lindahl, B. Hess, and D. van der Spoel. Gromacs 3.0: A package for molecular simulation and trajectory analysis. J. Mol. Mod., 7:306-317, 2001.

10. H. J. C. Berendsen, D. van der Spoel, and R. van Drunen. Gromacs: A messagepassing parallel molecular dynamics implementation. Comp. Phys. Comm., 91:4356, 1995.

11. M. W. Schmidt, K. K. Baldridge, J. A. Boatz, S. T. Elbert, M. S. Gordon, J. H. Jensen, S. Koseki, N. Matsunaga, K. A. Nguyen, S. J. Su, T. L. Windus, M. Dupuis, and J. A. Montgomery. Gamess program package. J. Comput. Chem., 14:1347-1363, 1993.

12. H. J. C. Berendsen, J. P. M. Postma, A. DiNola, and J. R. Haak. Molecular dynamics with coupling to an external bath. J. Chem. Phys., 81:3684-3690, 1984.

13. S. Miyamoto and P. A. Kollman. Settle: An analytical version of the shake and rattle algorithms for rigid water models. J. Comp. Chem., 13:952-962, 1992.

14. U. Essman, L. Perela, M. L. Berkowitz, T. Darden, H. Lee, and L. G. Pedersen. A smooth particle mesh ewald method. J. Chem. Phys., 103:8577-8592, 1995. 